\title{
Feaseability and safety of laser turbinoplasty with partial bone resection in obstructive sleep apnea syndrome (OSAS)
}

\begin{abstract}
Introduction: One of the main causes of nasal blockage is the inferior nasal turbinates hypertrophy, with impact in sleep quality and obstructive sleep apnea symdrom treatment. The use of laser, when properly indicated by the appropriate wavelength, exhibits good tissue interaction for the use in the nasal mucosa, allowing to reduce the turbinates through minimally invasive procedures.
\end{abstract}

Objective: To describe a laser turbinoplasty technique, with partial bone removal, used to treat the hypertrophy of the inferior nasal turbinates in OSAS patients, demonstrating its feasibility and safety.

\begin{abstract}
Method: We retrospectively analised data from electronic medical records of patients submited to this procedure to treat the inferior nasal turbinates hipertrophy, from January 2009 to December 2013. The inclusion criteria was OSAS, diagnosed by polysonography, with age above 18years and followed for at least 12 weeks after the procedutre. Were excluded patients without the apropriate posoperative follow-up. By presenting a wavelength indicated for nasal surgery, was used the Ho:YAG laser, with 5Watts of power and frequency of $5 \mathrm{~Hz}$.
\end{abstract}

Results: Were included 112 OSAS patients, 59 (53\%) mild and $53(47 \%)$ moderate and severe, age ranging from 18 to 74, average of 38years. The proportion between man and woman was 3:1. The mean energy delivered was 1406 Joules for each nasal turbinate, always pulsed mode, with average time of 12 minutes in each nostril. Nasal packing was not used in any case and patients in average were discharged twelve hours after the procedure. In $2 \%$ of cases there was increased secretion in the posoperative follow-up, controlled with oral antibiotic and nasal irrigation with hypertonic solution. This symptom did not persist for more than 4 weeks in any case. None complication considered severe occurred.

Conclusion: The technique of laser turbinoplasty presented enable a procedure with little damage to nasal mucosa and low complication rate, demonstrating clinically its feasibility and safety in OSAS patients, what can contribute to increase the Mandibular Advancement Splint (MAS) and CPAP compliance.

Keywords: laser turbinoplasty technique, sleep apnea, mandibular advancement splint, continuous positive airway pressure, rhinorrhea, osteomyelitis
Volume 4 Issue I - 2016

\author{
Eric Thuler \\ Hospital Samaritano, Brazil
}

Correspondence: Eric Thuler, Hospital Samaritano, Conselheiro Brotero Street I505, Brazil, Tel 55I I9922 I3455, Email erthuler@gmail.com

Received: July 13,2016 | Published: February 18, 2016
Abbreviations: MAS, mandibular advancement splint; CPAP, continuous positive airway pressure; AHI, apnea hipopnea index

\section{Introduction}

The nose is the airway gateway, therefore, nasal pathologies interfere directly in respiratory resistance, ${ }^{1,2}$ with possible impact on the pathophysiology of sleep apnea ${ }^{3,4}$ and its treatment with Mandibular Advancement Splint (MAS) and Continuous Positive Airway Pressure (CPAP).

One of the most prevalent nasal pathologie is rhinitis, where characteristically occurs inflammation of the nasal mucosa, resulting in sneezing, rhinorrhea and nasal blockage. Chronic conditions may have a poor response to drug treatment, mainly when the inferior nasal turbinate mucosa is hyperplastic or there is hypertrophy of the turbinate bone. ${ }^{5}$

In these cases, surgical procedures to reduce the volume of the inferior nasal turbinate may be necessary, where turbinectomies and turbinoplasties are included. ${ }^{6}$
The turbinectomy can be partial or total and involve, respectively, resection of part or all the nasal turbinate. Currently, resections has fallen into disuse due to impairment of nasal physiology and high risk of postoperative complications, mainly in OSAS patients.

The turbinoplasty with submucosal bone ressection allows greater preservation of the nasal turbinate, removing only a small portion of the bone structure, usually with the aid of a microscope or endoscope.

Both procedures typically have the risk of bleeding and are followed by nasal packing to prevent excessive blood loss, what can increase the risk of postoperative complications in severe OSAS patients with oxygen dessaturation. ${ }^{7}$

There are turbinoplasties discrebed just removing the inferior turbinate mucosa hypertrophied, in most of the cases inducing necrosis and tissue resorption, with reduction of tissue volume through the secondary cicatricial retraction. It can be done with conventional electrocautery, devices with controlled temperature (Somnoplasty, Coblation), surgical lasers and microdebriders. ${ }^{8}$ 
However, they are less effective to reduce de turbinate volume and may occur excessive epitelial destruction, with bone exposure and increased formation of crusts and secretions postoperatively. ${ }^{9}$

In general, the major complications of these procedures are bleeding, turbinate excessive resection causing atrophic rhinitis, and infections, especially the turbinate osteomyelitis. ${ }^{10}$

Recurrence of turbinate hypertrophy is common, especially in procedures designed only to reduce the mucosa. Evidence indicates that occur on average two years after surgery, with recurrence of obstructive symptoms, especially in allergic patients.

The surgical laser was introduced to treat the inferior nasal turbinate hipertrophy in an attempt to reduce the risks of bleeding and allow outpatient procedure, but mainly used to vaporize the mucosa. ${ }^{11}$

The laser physical properties finds the ideal characteristics to perform a turbinoplasty with submucosal partial bone ressection, specially in the surgical treatment of nasal blockage due to the inferior nasal turbinate hypertrophy in OSAS patients. However, its feasibility and safety was not described before.

\section{Objectives}

The aim of this manuscript is to describe a laser turbinoplasty technique with submucosal partial bone ressection, to treat the inferior nasal turbinate hipertrophy in OSAS patients, demonstrating its feasibility and safety.

\section{Materials and methods}

Were analyzed retrospectively the data from electronic medical records of patients submitted to laser turbinoplasty from January 2009 to December 2013, with approval from the hospital research ethics committee (NP 268.734).

This procedure was indicated to treat nasal blockage, unresponsive to medical treatment, secundary to hypertrophy of the inferior nasal turbinate confirmed by CT scan.

The inclusion criteria was patients with age above 18years and OSAS, diagnosed by polysonography according to the American Academy of Sleep Medicine manual, considering the Apnea Hipopnea Index (AHI) below 15 as mild, above 15 and below 30 moderate and above 30 severe.

Were excluded patients that was not diagnosed with OSAS and with nasal blockage that was not associated to hipertrophy of the inferior nasal turbinate or were not rightly followed during postoperative period.

Based on physical interaction with the nasal mucosa ${ }^{12}$ we choose the Ho:YAG laser (Dornier, Germany) that has a wavelength of $2100 \mathrm{~nm}$ and operate in pulsatile mode. To minimize the termal demage, we choose a low frequency $(05 \mathrm{~Hz})$ and power (5Watts), delivering 1.0 joules per pulse.

Our technique of turbinoplasty starts with a longitudinal laser incision at the lower bord of the the inferior nasal turbinate bone, followed by careful subperiosteal dissection and resection of a small portion of the bone. Finally the mucosa is repositioned with preservation of its integrity, using the laser for welding the edges of the incision, without the use of hemostatic substances or nasal packing.

All procedures were performed under intravenous anestesia, using propofol, with the aid of Odegree nasal endoscope, coupled to a video system. The laser fiber was coupled to a thin, angled tip, allowing more control and precision in the incision along the inferior nasal turbinate.

The patients remained in Day Hospital unit and were discharged, on average, twelve hours after the procedure, with no need of hospitalization, receiving nasal vasoconstrictor drops (oxymetazoline) and oral antibiotic (cephalexin) in the first five days of the postoperative period. As in this period may occur minimal blood exit at efforts, rest was recommended to all patients.

The patients returned for postoperative follow-up with five days, two weeks, four weeks, eight weeks and twelve weeks after the procedure. In the first and second returns was performed aspiration of the nasal cavity to remove secretion, without much concern with the crusts in the site of the turbinoplasty incision, and nasal irrigation was recommended with hypertonic saline solution ( $3 \%$ Sodium Chloride) during this period.

\section{Results}

During the period analised were submitted to this procedure 558 patients, where 112 met the inclusion criteria, 59 (53\%) mild OSAS and $53(47 \%)$ moderate and severe OSAS, age ranging from 18 to 74 , average of 38 years. The proportion between man and woman was $3: 1$.

In mild OSAS, the procedure was mainly indicated to improve the use of oral apliance. In moderate and severe OSAS the procedure was proposed to improve CPAP compliance, when nasal blockage was supposedly interfering in CPAP adherence.

The amount of energy applied ranged from 790 to $2271 \mathrm{~J}$, averaging 1406 Joules at each inferior nasal turbinate, with time ranging from 6 to 18 minutes, average of 12 minutes in each nostril.

In the first postoperative return, was observed mild edema of the inferior turbinate, leading to nasal congestion, with complete resolution at the next return, two weeks after the surgery, when severe OSAS patients were than recomended to return the CPAP use, with oronasal mask.

With the recommended postoperative care with the use of hypertonic saline solution for nasal irrigation, the crusts were removed in all cases within four weeks. After this period, the transition to nasal mask was achieved in most of CPAP users $(92,5 \%)$, except in four cases $(7,5 \%)$.

Infection occurred in $2 \%$ of the cases, between the second and fourth week, with complaining of increased secretion, resolved using oral antibiotic (Amoxacilin) for 7 (Seven)days.

In 1 case $(0,9 \%)$ there was a mild bleeding, after 10days of the procedure, easily controlled with local measures. Nasal synechiae didn't occurred in any case.

There was no major complications, such as severe bleeding, persistent crust formation, amendment of smell or atrophic rhinitis with empty nose syndrome.

\section{Discussion}

Considering the airway as a flexible tube and the Starling model, the obstruction at its entry (nose) would result in a higher intraluminal negative pressure (pharynx), increasing the collapse. ${ }^{1,2}$ In case of mouth breathing, there is a 2.5 adicional increase in resistance to the air inlet, ${ }^{3}$ caused by the posterior displacement of the tongue, secundary to mouth opening and mandibular rotation. ${ }^{4}$ 
For those reasons, treat the nasal blockage in OSAS patients certainly contribute to tretament, reducing the airway resistance, the pharyngeal collapse and the mouth breathing, helping to MAS and CPAP compliance. ${ }^{13}$ The contribution for CPAP compliance is even better to allow the use of nasal masks, where positive pressure can easily reach the pharyngeal space.

A revision of different techniques to treat the inferior turbinate hipertrophy concluded that the ideal procedure aims volume reduction with preservation of functional mucosa, pointing turbinoplasty with submucosal resection the procedure of choice. ${ }^{5,6}$

Randomized studies also demonstrated that the turbinoplasty has better results, enabling clinical improvement and preservation of the mucosa. Major complications occured with partial turbinectomy, with excessive crusting formation, bleeding and synechiae. ${ }^{8,9}$

Looking for laser surgery, ${ }^{14}$ the most appropriate lasers for the use in nasal turbinate are Holmium: Yttrium Aluminum Garnet (Ho:YAG) and Diode Laser. What differs each type of laser is the wavelength of the emitted light beam, determining its penetration depth and tissue interaction. ${ }^{15}$

In a electron microscopy study comparing different forms of surgical lasers in the inferior nasal turbinate ${ }^{16}$, the Ho:YAG laser showed good hemostasis with low penetration and minimal charring. Analising the complications using the Ho:YAG Laser, ${ }^{17,18}$ there were $5 \%$ of minor bleeding, with crust formation during two weeks. Long term results, observed by acoustic rhinomanometry and subjective evaluation three years after the procedure, demonstrated a significant increase in nasal airflow in $67 \%$ of patients.

In most of manuscripts published using laser to reduce the inferior nasal turbinate, ${ }^{17,19-27}$ the laser was not used reggarding the OSAS treatment and was applied to vaporize the mucosa with epithelial destruction, increasing crust formation and the risk of posoperative complications.

In the present technique, the laser was used in OSAS patients to make just a incision in the inferior turbinate, using the pulsatile mode in a low frequency, causing less thermal damage to adjacent tissue. ${ }^{28}$ Removing a portion of the nasal turbinate bone already provides volume reduction and airspace gain, with a greatter preservation of the mucosa, contributing to a better healing process and reducing the complications risk.

Generally, patients report an improvement in nasal breathing in few days after the procedure, possibly explained by the reasons cited above, helping the early return to OSAS treatment (MAS or CPAP).

Another importante issue to mention is the severe OSAS, where many patients already had cardiovascular consequences of the disease, using antihypertensive drugs, aspirin and anticoagulants. Even in this group was possible to avoid the nasal package, without any case of bleeding or other complications in the posoperative period.

However, in the present data we could not get any objective measure about CPAP or MAS compliance and we can not assert that this procedure improve OSAS treatment. Further analisys with objective mesures is necessary to evaluate the real gain in compliance, determining its real contribution in OSAS treatment.

\section{Conclusion}

The technique of laser turbinoplasty presented enable a procedure with little damage to nasal mucosa and low complication rate, demonstrating clinically its feasibility and safety in OSAS patients, what may contribute for MAS and CPAP compliance.

\section{Acknowledgments}

None.

\section{Conflicts of interest}

Author declares there are no conflicts of interest.

\section{Funding}

None.

\section{References}

1. Pevernagie D, De Meyer MM, Claeys S. Sleep, breathing and the nose. Sleep Med Rev. 2005;9(6):437-451.

2. Fitzpatrick MF, McLean H, Urton AM, et al. Effect of nasal or oral breathing route on upper airway resistance during sleep. Eur Respir J. 2003;22(5):827-832.

3. Georgalas C. The role of the nose in snoring and obstructive sleep apnoea: An update. Eur Arch Oto-Rhino-Laryngology. 2011;268(9):1365-1373.

4. Lee SH, Choi JH, Shin C, et al. How does open-mouth breathing influence upper airway anatomy? Laryngoscope. 2007;117(6):1102-1106.

5. Uzun L, Ugur MB, Savranlar A, et al. Classification of the inferior turbinate bones: A computed tomography study. Eur J Radiol. 2004;51(3):241-245.

6. Hol MKS, Huizing EH. Treatment of inferior turbinate pathology: A review and critical evaluation of the different techniques. Rhinology. 2000;38(4):157-166.

7. Passàli D, Passàli FM, Damiani V, et al. Treatment of inferior turbinate hypertrophy: A randomized clinical trial. Ann Otol Rhinol Laryngol. 2003;112(8):683-688.

8. Chen YL, Liu CM, Huang HM. Comparison of microdebriderassisted inferior turbinoplasty and submucosal resection for children with hypertrophic inferior turbinates. Int $J$ Pediatr Otorhinolaryngol. 2007;71(6):921-927.

9. Passàli D, Anselmi M, Lauriello M, et al. Treatment of hypertrophy of the inferior turbinate: Long-term results in 382 patients randomly assigned to therapy. Ann Otol Rhinol Laryngol. 1999;108(6):569-575.

10. Scheithauer MO. Surgery of the turbinates and "empty nose" syndrome. Laryngorhinootologie. 2010;89(Suppl 1):S79-S102.

11. Serrano E. The Holmium: Yag laser for treatment of the inferior turbinate hypertrophy. Rhinology. 1998;36(2):77-88.

12. Welch AJ, Torres JH, Cheong WF. Laser physics and laser-tissue interaction. Tex Heart Inst J. 1989;16(3):141-149.

13. Park CY, Hong JH, Lee JH, et al. Clinical effect of surgical correction for nasal pathology on the treatment of obstructive sleep apnea syndrome. PLoS One. 2014;9(6): e98765.

14. Milonni PW, Eberly JH. Laser Physics. Laser Phys. 2010;61(9):961-964.

15. Cox B. Introduction to Laser-Tissue Interactions. 2007.

16. Janda P, Sroka R, Betz CS, et al. Comparison of laser induced effects on hyperplastic inferior nasal turbinates by means of scanning electron microscopy. Lasers Surg Med. 2002;30(1):31-39.

17. Serrano E, Percodani J, Yardeni E, et al. The Holmium: YAG laser for treatment of inferior turbinate hypertrophy. Rhinology. 1998;36(2):77-80. 
18. Leunig A, Janda P, Sroka R, et al. Ho: YAG laser treatment of hyperplastic inferior nasal turbinates. Laryngoscope. 1999;109(10):1690-1695.

19. Janda P, Sroka R, Betz CS, et al. Ho: YAG and diode laser treatment of hyperplastic inferior nasal turbinates. . 2002;81(7):484-490.

20. Lippert BM, Werner JA. Long-term results after laser turbinectomy. Lasers Surg Med. 1998;22(2):126-134.

21. Caffier PP, Scherer H, Neumann K, et al. Diode laser treatment in therapy-resistant allergic rhinitis: Impact on nasal obstruction and associated symptoms. Lasers Med Sci. 2011;26(1):57-67.

22. Rejali SD, Upile T, McLellan D, et al. Inferior Turbinate Reduction in Children Using Holmium YAG Laser - A Clinical and Histological Study. Lasers Surg Med. 2004;34(4): 310-314.

23. Selkin SG. Laser turbinectomy as an adjunct to rhinoseptoplasty. Arch Otolaryngol. 1985;111(7):446-449.
24. Englender M. Nasal laser mucotomy (L-mucotomy) of the interior turbinates. J Laryngol Otol. 1995;109(4):296-299.

25. Fukutake T, Yamashita T, Tomoda K, et al. Laser surgery for allergic rhinitis. Arch Otolaryngol neck Surg. 1986;112(4):756-761.

26. Cook JA, McCombe AW, Jones AS. Laser treatment of rhinitis-1 year follow-up. Clin Otolaryngol Allied Sci. 2007;18(3):209-211.

27. Galletta A, Amato G. Photocoagulation of the hypertrophic lower turbinates using ND:YAG laser: functional results. Acta Otorhinolaryngol Ital. 1997;17(5):329-338.

28. Geldi C, Bozkulak O, Tabakoglu HO, et al. Development of a surgical diode-laser system: controlling the mode of operation. Photomed Laser Surg. 2006;24(6):723-729. 